VLSI DESIGN

1998, Vol. 8, Nos. (1-4), pp. 215-217

Reprints available directly from the publisher

Photocopying permitted by license only
(C) 1998 OPA (Overseas Publishers Association) N.V.

Published by license under the Gordon and Breach Science Publishers imprint. Printed in India.

\title{
Phase Space Boundary Conditions and Quantum Device Transport
}

\author{
H. L. GRUBIN ${ }^{\mathrm{a}, *}$, J. R. CASPAR ${ }^{\mathrm{a}}$ and D. K. FERRY ${ }^{\mathrm{b}}$ \\ ${ }^{a}$ Scientific Research Associates, Inc., P. O. Box 1058 Glastonbury, CT 06033; \\ ${ }^{\mathrm{b}}$ Center for Solid State Electronics Research, Arizona State University, Tempe AZ 85287
}

\begin{abstract}
Device-device interaction is described within the framework of the quantum Liouville equation. Approximations to treating this interaction, within the framework of alterations to phase space boundary conditions, are illustrated.
\end{abstract}

Keywords: Liouville equation, phase space boundary conditions, quantum devices, thermal deBroglie wavelength, device-device interactions

\section{INTRODUCTION}

Classical descriptions of device-device interactions involve simple boundary conditions, often through an external circuit, or in some cases through electromagnetic interactions. Quantum device-device interactions are richer, as there are several critical lengths. Device-device interactions on a scale of, or smaller than, a thermal deBroglie wavelength, $\lambda_{\text {DeBroglie }}=\sqrt{\hbar^{2} / 2 m k_{B} T}$, introduce coherence, and two barriers separated by distances of the order of $\lambda_{\text {deBroglie }}$ result in resonant tunneling. Debye length-scales are a second critical length. But the critical length-scale of interest is an inter-device length, $\lambda_{\text {inter-device}}$, which is the minimum inter-device separation before changes in one device affect the properties of an adjacent device.
The concept of an inter-device separation is more general than that associated with either the thermal deBroglie wavelength or the Debye length, as its value arises from phase space boundaries and involves at least two coordinates. In this paper we illustrate the concept of an inter-device length for a pair of double barriers.

We first examine the form of the Liouville potential in the coordinate representation: $V\left(x, x^{\prime}\right)$ $\equiv V\left(x-V x^{\prime}\right), x$ and $x^{\prime}$ being phase space coordinates. Based on the form of $V\left(x, x^{\prime}\right)$ we identify both non-interacting and interacting diodes. We then solve the quantum Liouville equation for a surrogate problem where changes in the charge distribution arise from changes in the phase space boundary conditions that, we argue, represent the device-device interaction.

\footnotetext{
* Corresponding author
} 
The case of non-interacting diodes is identified in Figure 1, where we display $V\left(x, x^{\prime}\right)$ for a pair of double barrier structures. The coordinate divisions represent multiples of an isolated device, and for a double barrier RTD of length $200 \mathrm{~nm}$, the number 2 corresponds to $400 \mathrm{~nm}$. In the following we will refer to two regions: region 1 , where $0 \leq x \leq 1,0$ $\leq x^{\prime} \leq 1$, and region $3: 1 \leq x \leq 2,1 \leq x^{\prime} \leq 2$. The pairs of double barriers are centered in regions 1 and 3 , and the absence of any curvature away from the double barrier Liouville potential indicates that these two structures are not interacting. Figure 2 shows a modification of the Liouville potential for a perturbation centered on the diagonal in region 1 at a point $x=x^{\prime}=0.75$. The distortion, which spills into region ' 3 ', as shown in Figure 3, will alter the density matrix in region 3. On the basis of Figure 3 and diagrams similar to it, we argue that introducing variations in the phase space boundary conditions can approximate the effect of device-device interaction on the behavior of any given device.

\section{THE ROLE OF PHASE SPACE BOUNDARY CONDITIONS AND CONCLUSIONS}

While solutions to the quantum Liouville equation provide the means for examining device-device

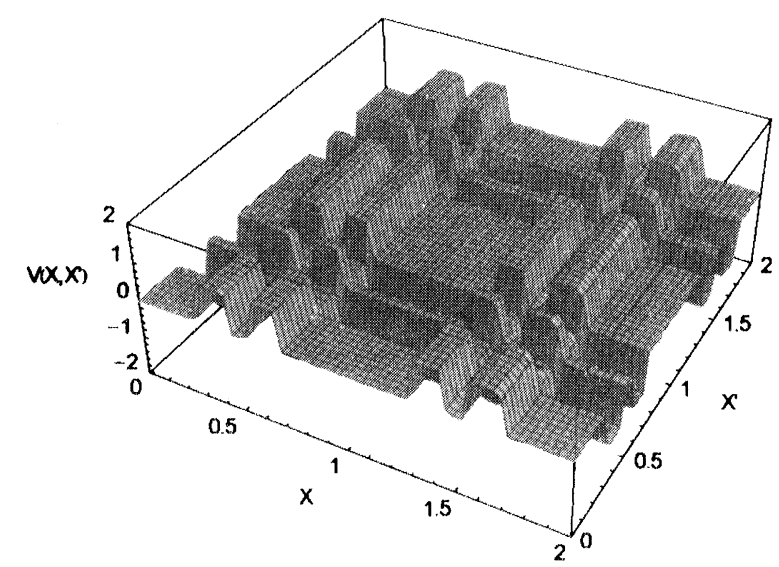

FIGURE 1 The Liouville potential for a pair of noninteracting double barrier diodes centered in regions 1 and 3 .

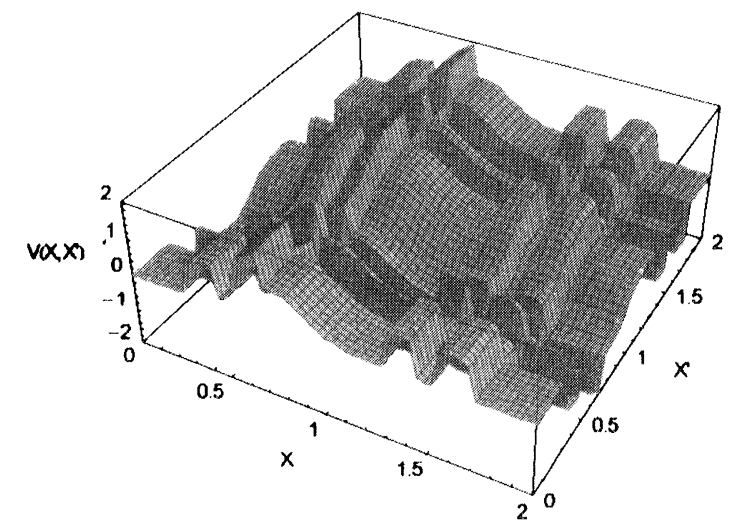

FIGURE 2 The Liouville potential for a perturbation in region 1 , that spills over into region 3 . These structures are interacting.

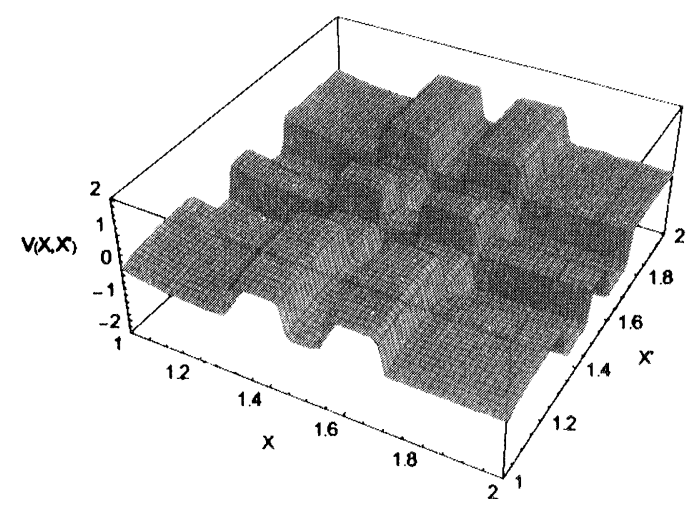

FIGURE 3 A display, within region 3, of a section of the distorted-Liouville potential of Figure 2. Note the distortion of the Liouville potential near the origin of region 3 .

interactions on a large scale, as a practical matter this approach is premature, as computational times would be excessive. Rather, we solve the Liouville equation subject to boundary conditions that represent the effects of device-device interaction. In particular we have solved the quantum Liouville equation [1]:

$$
\begin{aligned}
& \left.i \hbar\left(\frac{\partial \rho\left(x, x^{\prime}\right)}{\partial t}+\frac{\partial \rho\left(x, x^{\prime}\right)}{\partial t}\right]_{\text {dissipation }}\right) \\
& =-\frac{\hbar^{2}}{2 m}\left(\frac{\partial^{2}}{\partial x^{2}}-\frac{\partial^{2}}{\partial x^{\prime 2}}\right) \rho\left(x, x^{\prime}\right)+V\left(x, x^{\prime}\right) \rho\left(x, x^{\prime}\right),
\end{aligned}
$$


for a $200 \mathrm{~nm}$ square region for symmetric and asymmetric double barrier structures, and symmetric quantum wells. For noninteracting structures the density matrix boundary conditions along the line $x^{\prime}=200 \mathrm{~nm}$, was $\exp \left[-\left(\left(x-x^{\prime}\right) / 4\right.\right.$ $\left.\left.\lambda_{\text {deBroglie }}\right)^{2}\right]$. For calculations representing interacting structures the boundary condition is $A\left(x-x^{\prime}\right)$ $\exp \left[-\left(\left(x-x^{\prime}\right) / 4 \lambda_{\text {deBroglie }}\right)^{2}\right]$. The modulating function was chosen arbitrarily. Figure 4 displays the boundary variation invoked in this study, and Figure 5 displays the effects of this boundary variation on the charge density within the symmetric RTD. Figure 5 also displays the potential energy. The calculation with the smallest charge within the barrier occurs for a modulating function that is set to unity. For the change in the modulating function shown in Figure 4, there is significant charge accumulating within the barriers. Very similar results emerge from the asymmetric double barrier structures. Significantly, the form of the perturbation affects the form of the

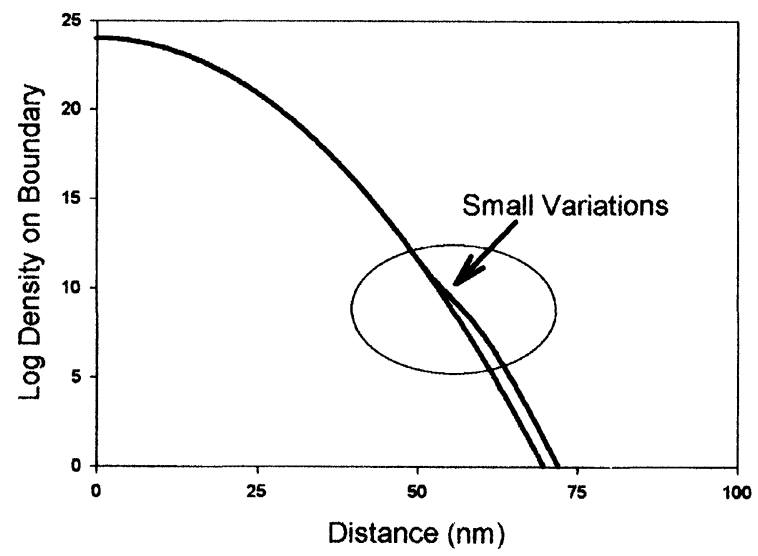

FIGURE 4 Variations of the density matrix boundary condition along $x^{\prime}=200 \mathrm{~nm}$ for a double barrier structure.

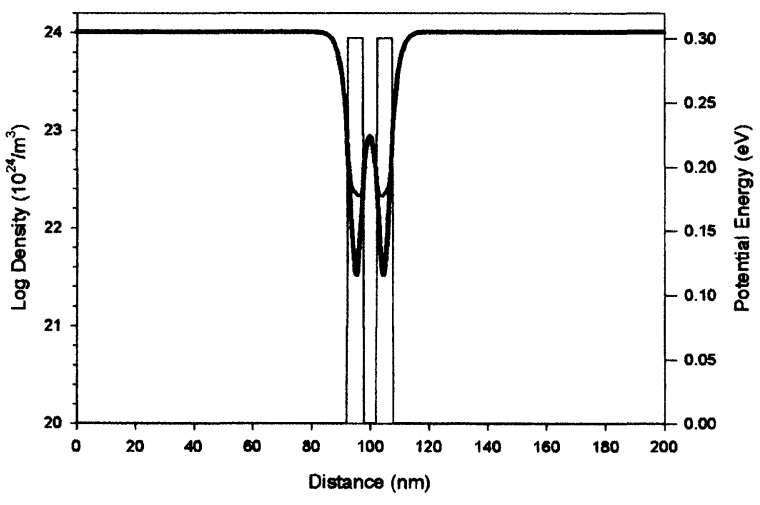

FIGURE 5 The density distributions for a non-self consistent solution to the quantum Liouville equation subject to the variations of Figure 4.

charge density alterations. Additional calculations reveal that the effect of the boundary is most dramatic for perturbations close to the origin. Because of the Gaussian decay of the boundary condition, the further the perturbation is away from the origin the weaker the effect. The results also suggest that the shorter the inter-device separation the greater the interaction. Supplementary calculations indicate that double barrier diodes with cladding regions doped to $10^{24} / \mathrm{m}^{3}$, require a minimum separation of approximately $180 \mathrm{~nm}$ to be considered as isolated.

\section{Acknowledgements}

This work was sponsored by DARPA.

\section{References}

[1] Grubin, H. L. (1994). Density Matrix Simulations of Semiconductor Devices: "Quantum Transport in Ultra Small Devices", Eds. D. K. Ferry, H. L. Grubin and A. P. Jauho, Plenum Press, N. Y. 

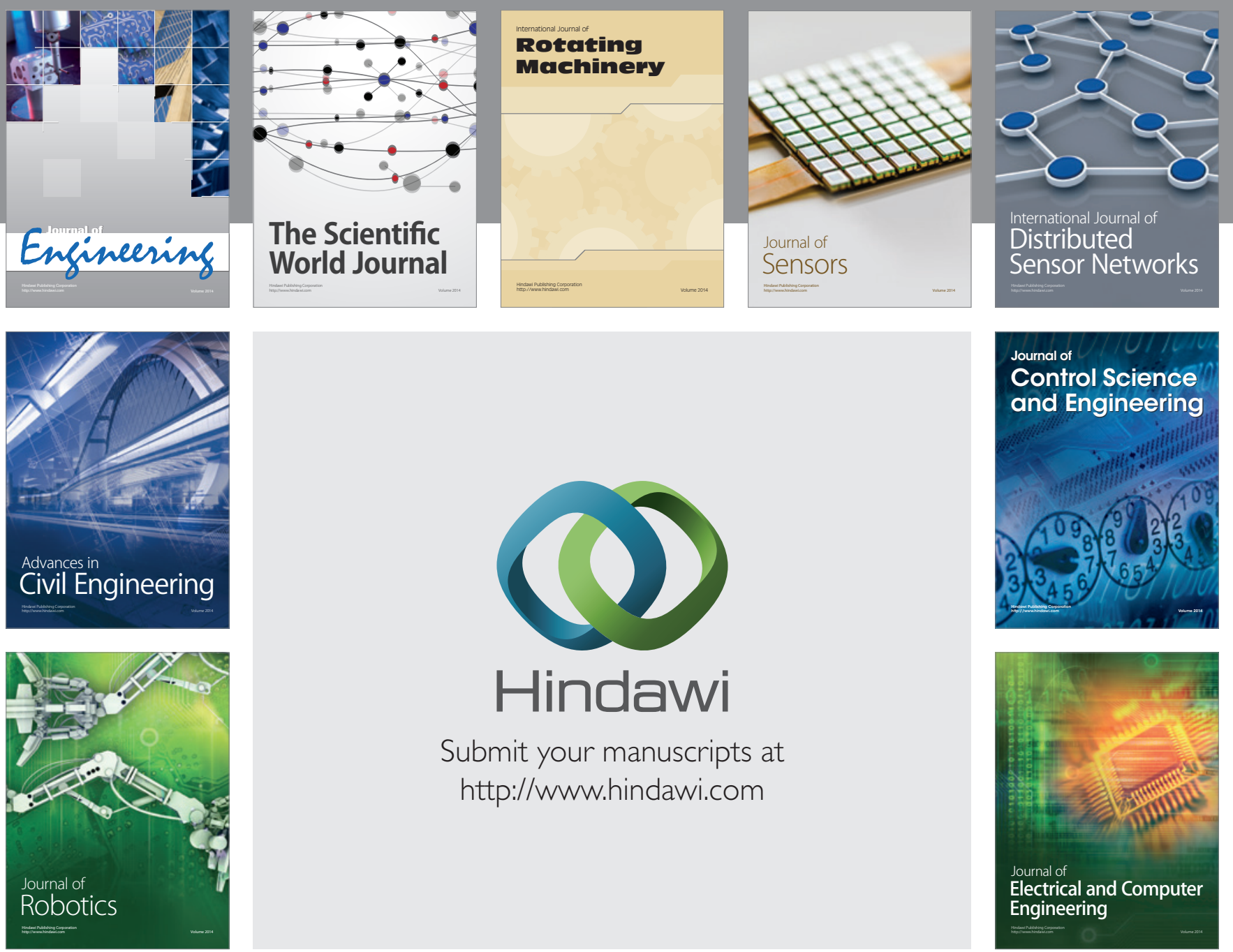

Submit your manuscripts at

http://www.hindawi.com
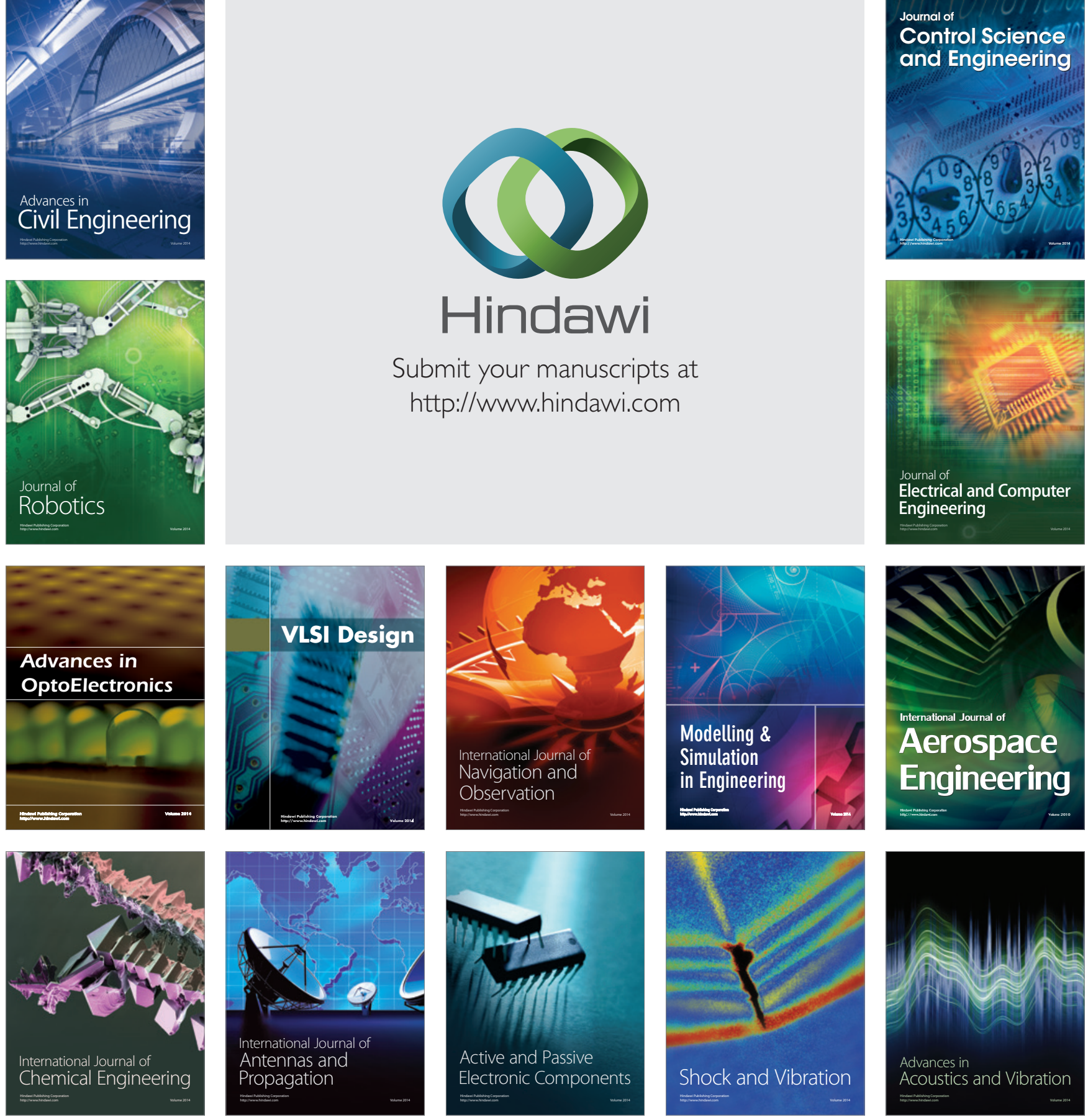